\title{
Health-related quality of life and physical activity in children with inherited cardiac arrhythmia or inherited cardiomyopathy: the prospective multicentre controlled QUALIMYORYTHM study rationale, design and methods
}

Pascal Amedro ${ }^{1,2,3^{*}}$ (D), Oscar Werner ${ }^{4}$, Hamouda Abassi ${ }^{4,15}$, Aymeric Boisson ${ }^{4}$, Luc Souilla ${ }^{4}$, Sophie Guillaumont ${ }^{4,5}$, Johanna Calderon ${ }^{4,15}$, Anne Requirand ${ }^{4}$, Marie Vincenti ${ }^{4,15}$, Victor Pommier ${ }^{4,5}$, Stefan Matecki ${ }^{4,15}$, Gregoire De La Villeon ${ }^{4,5}$, Kathleen Lavastre ${ }^{4}$, Alain Lacampagne ${ }^{15}$, Marie-Christine Picot ${ }^{6}$, Constance Beyler ${ }^{7}$, Christophe Delclaux $^{7}$, Yves Dulac $^{8}$, Aitor Guitarte ${ }^{8}$, Philippe Charron ${ }^{9}$, Isabelle Denjoy-Urbain ${ }^{9}$, Vincent Probst ${ }^{10}$, Alban-Elouen Baruteau ${ }^{11}$, Philippe Chevalier ${ }^{12}$, Sylvie Di Filippo ${ }^{12}$, Jean-Benoit Thambo 1,2,3, Damien Bonnet ${ }^{13}$ and Jean-Luc Pasquie ${ }^{14,15}$

\begin{abstract}
Background: Advances in paediatric cardiology have improved the prognosis of children with inherited cardiac disorders. However, health-related quality of life (QoL) and physical activity have been scarcely analysed in children with inherited cardiac arrhythmia or inherited cardiomyopathy. Moreover, current guidelines on the eligibility of young athletes with inherited cardiac disorders for sports participation mainly rely on expert opinions and remain controversial.

Methods: The QUALIMYORYTHM trial is a multicentre observational controlled study. The main objective is to compare the QoL of children aged 6 to 17 years old with inherited cardiac arrhythmia (long QT syndrome, Brugada syndrome, catecholaminergic polymorphic ventricular tachycardia, or arrhythmogenic right ventricular dysplasia), or inherited cardiomyopathy (hypertrophic, dilated, or restrictive cardiomyopathy), to that of age and gender-matched healthy subjects. The secondary objective is to assess their QoL according to the disease's clinical and genetic characteristics, the level of physical activity and motivation for sports, the exercise capacity, and the socio-demographic data. Participants will wear a fitness tracker (ActiGraph GT3X accelerometer) for 2 weeks. A total of 214 children are required to observe a significant difference of $7 \pm 15$ points in the PedsQL, with a power of $90 \%$ and an alpha risk of $5 \%$.
\end{abstract}

\footnotetext{
*Correspondence: pascal.amedro@gmail.com

1 Department of Paediatric and Adult Congenital Cardiology, M3C

National Reference Centre, Haut-Lévêque Cardiology Hospital, Bordeaux

University Hospital, Avenue de Magellan, 33604 Pessac Cedex, France

Full list of author information is available at the end of the article
}

(c) The Author(s) 2021. Open Access This article is licensed under a Creative Commons Attribution 4.0 International License, which permits use, sharing, adaptation, distribution and reproduction in any medium or format, as long as you give appropriate credit to the original author(s) and the source, provide a link to the Creative Commons licence, and indicate if changes were made. The images or other third party material in this article are included in the article's Creative Commons licence, unless indicated otherwise in a credit line to the material. If material is not included in the article's Creative Commons licence and your intended use is not permitted by statutory regulation or exceeds the permitted use, you will need to obtain permission directly from the copyright holder. To view a copy of this licence, visit http://creativecommons.org/licenses/by/4.0/. The Creative Commons Public Domain Dedication waiver (http://creativeco mmons.org/publicdomain/zero/1.0/) applies to the data made available in this article, unless otherwise stated in a credit line to the data. 
Discussion: After focusing on the survival in children with inherited cardiac disorders, current research is expanding to patient-reported outcomes and secondary prevention. The QUALIMYORYTHM trial intends to improve the level of evidence for future guidelines on sports eligibility in this population.

Trial registration ClinicalTrials.gov Identifier: NCT04712136, registered on January 15th, 2021 (https://clinicaltrials.gov/ ct2/show/NCT04712136).

Keywords: Quality of life, Physical activity, Paediatrics, Inherited cardiac arrhythmia, Genetic cardiomyopathy

\section{Background}

Prognosis of children with congenital cardiac disorders has significantly improved in the past two decades, due to great advances in prenatal screening, intensive neonatal care and modern invasive therapies [1]. As a result, more attention has been recently given to health-related quality of life (QoL) and secondary prevention in paediatric cardiology $[2,3]$.

Traditionally, congenital cardiac disorders are divided into 3 groups: congenital heart disease (CHD) for structural cardiac anomalies, inherited cardiac arrhythmia and inherited cardiomyopathy. So far, QoL studies in paediatric cardiology have mainly focused on the group of children with CHD and, although the overall level of QoL is good in this population, our group highlighted the existing correlation between exercise capacity and QoL, and the risk for physical deconditioning, even for non-serious CHD $[4,5]$. Indeed, despite the promotion of physical activity in the current guidelines [6-8], many children with CHD experience social barriers to physical activity and "remain on the side-line" at school or in their social life [9]. Ultimately, these patients may be exposed to the consequences of a sedentary lifestyle: overweight, high blood pressure, social exclusion, and impaired QoL [10-12]. Conversely, patients with CHD who have been physically active since childhood are less likely to become sedentary adults [13].

Such consistent data are not available in the literature for children from the two other groups, e.g. inherited cardiac arrhythmia or inherited cardiomyopathy, for which QoL and physical activity have been rarely studied. Some heterogeneous data were provided from small paediatric [14-16] or adult $[17,18]$ cohorts, however no prospective controlled study has analysed the determinants of QoL and the level of physical activity in children with inherited cardiac disorders [19]. We hypothesized that QoL scores and physical activity levels in children with inherited cardiac disorders are reduced compared to the healthy subjects.

Therefore, the multicentre observational controlled QUALIMYORYTHM trial aims to evaluate the QoL, exercise capacity, and the level of physical activity of children with inherited cardiac arrhythmia or inherited cardiomyopathy, in comparison with a matched control population. This study will also evaluate the main factors associated with QoL in this population.

\section{Methods \\ Objectives}

The main objective of the QUALIMYORYTHM trial is to compare the QoL of children aged 6 to 17 years old with inherited cardiac arrhythmia or inherited cardiomyopathy to that of matched healthy children.

The secondary objective is to assess, in this population, the QoL according to the disease clinical and genetic characteristics, the level of physical activity, the exercise capacity, and the socio-demographic data.

\section{Study design}

The QUALIMYORYTHM trial is a prospective, multicentre controlled observational study with a follow-up of 2 weeks and a recruitment duration of 18 months. All screened subjects will be identifiable throughout the study by a unique subject number. The eligible patients will be divided into 2 groups (Fig. 1):

Group 1: children aged 6 to 17 years old with an inherited cardiac arrhythmia or an inherited cardiomyopathy.

Group 2: age and gender-matched control group, with healthy children aged 6 to 17 years old.

\section{Setting}

Patients will be recruited during a paediatric cardiology consultation among the French expert centres for inherited cardiac arrhythmia or an inherited cardiomyopathy (CARDIOGEN national network, http://www.filie re-cardiogen.fr). Overall, 7 university hospitals, labelled as expert centres in paediatric inherited cardiac disorders by health authorities, will participate in patient recruitment.

Conduct of the study will be led by the principal investigator (supported, when necessary, by the coinvestigators), a research fellow, and a clinical research coordinator, all of whom are trained in Good Clinical Practice and in the requirements of the study protocol.

\section{Study population}

Children aged of 6 to 17 years old with an inherited cardiac arrhythmia (long QT syndrome, Brugada syndrome, 

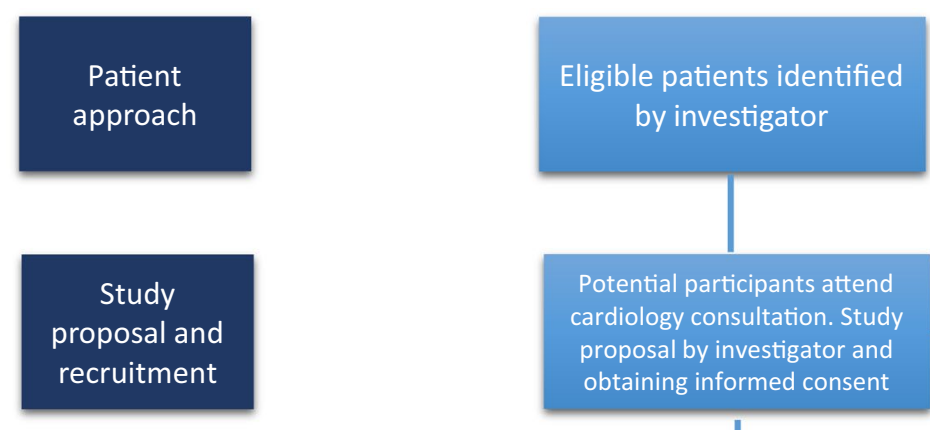

by investigator

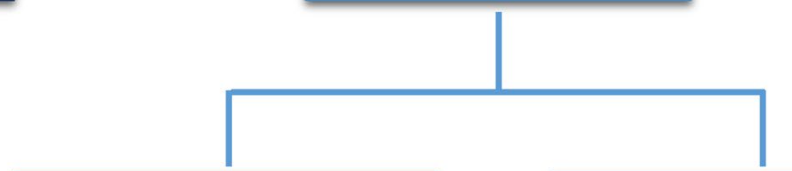

No response, negative response, ineligible patient

\section{Group $1(N=107)$}

6 to 17 y.o. children with

inherited cardiac arrhythmia

or inherited cardiomyopathy

Group $2(N=107)$

6 to 17 y.o. healthy children

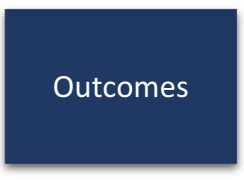

Quality of life

Exercise capacity (CPET)

Physical activity

\section{Follow-up \\ period \\ (2 weeks)}
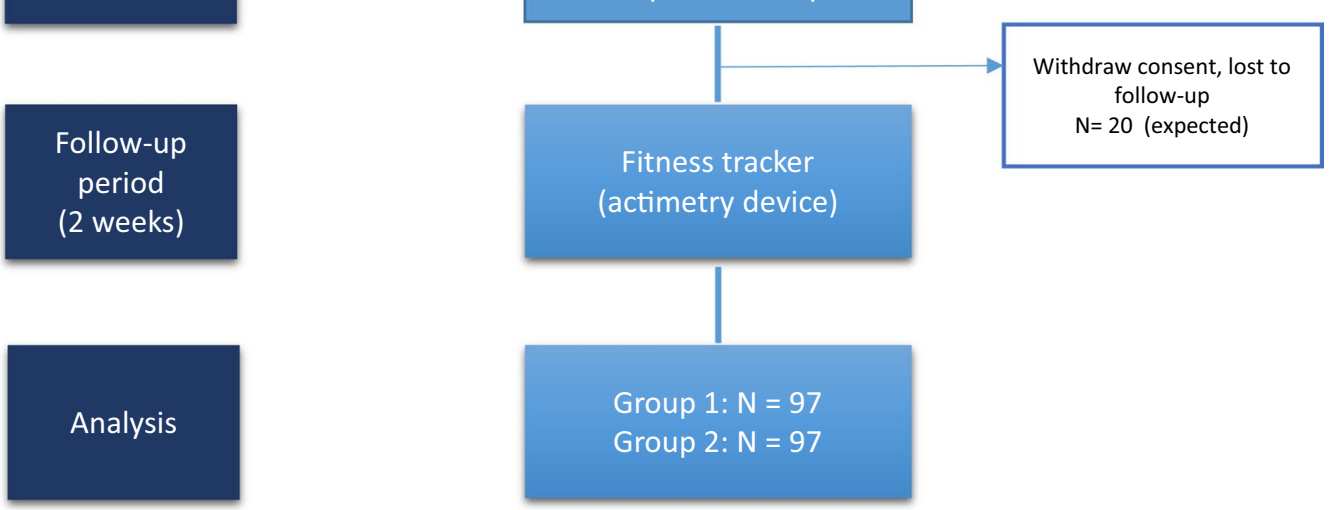

(actimetry device)

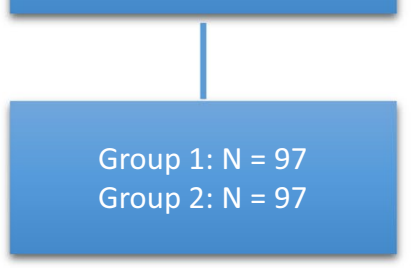

Fig. 1 Flow chart

catecholaminergic polymorphic ventricular tachycardia, or arrhythmogenic right ventricular dysplasia), or those with an inherited cardiomyopathy (hypertrophic, dilated, or restrictive cardiomyopathy), will be prospectively recruited during their annual follow-up.

The age- and gender-matched control group will be defined using the same method as in our previous paediatric exercise test studies [4, 5, 20-22]. Children aged 6 to 17 years old referred to the paediatric cardiology consultation for a non-severe functional symptom linked to exercise (murmur, palpitation, or dyspnoea) or for a medical sports certificate will be assessed for eligibility. These children will be classified in the control group only after a completely normal check-up, including physical examination, electrocardiogram, and echocardiography. Children with any chronic disease, medical condition (cardiac, neurologic, respiratory, muscular, or renal), or medical treatment and those requiring any further specialized medical consultation will not be eligible. 
Patients who are not able to answer the quality of life questionnaire (e.g. non-French speakers, severe mental illness) will not be eligible for the study. Detailed inclusion and exclusion criteria are reported in Table 1.

\section{Primary outcome}

The primary outcome is the total QoL score with the self-reported PedsQL questionnaire. The PedsQL questionnaire has four multidimensional scales: physical functioning (8 items), emotional functioning (5 items), social functioning (5 items), and school functioning (5 items). The three summary scores are: total scale score $(23$ items), physical health summary score (8 items), psychosocial health summary score ( 15 items). Each item uses a 5 -point Likert scale from 0 (never) to 4 (almost always). Items are reversed scored and linearly transformed to a 0-100 scale, higher scores indicating a better QoL. This instrument was validated by Varni et al. in healthy and patient populations and its psychometric properties showed reliability, validity and responsiveness to clinical change over time [23]. The translation and cultural adaptation into French was performed by MAPI Research Institute (www.mapi-trust.org), following the international guidelines [23] and this version showed good psychometric properties [24]. We recently performed the complete psychometric validation of the French self and proxy versions of the PedsQL generic questionnaire for 8 to 12 year-old children [25]. We also found a good sensitivity of the PedsQL in several controlled prospective

Table 1 Trial entry

\begin{tabular}{l}
\hline Inclusion criteria \\
\hline Male or female aged 6 to 17 years old \\
Group 1: Patients with an inherited cardiac arrhythmia (long QT \\
syndrome, Brugada syndrome, catecholaminergic polymorphic \\
ventricular tachycardia, or arrhythmogenic right ventricular dysplasia), \\
or those with an inherited cardiomyopathy (hypertrophic, dilated, or \\
restrictive cardiomyopathy) \\
Group 2: Children with a completely normal check-up, referred to \\
the paediatric cardiology consultation for a non-severe functional \\
symptom linked to exercise (murmur, palpitation, or dyspnoea) or for \\
a medical sports certificate \\
Informed consent of parents or legal guardians, and oral assent of \\
children \\
Exclusion criteria \\
Patients who are not able to understand or fill out the questionnaires \\
(QoL, physical activity and motivation questionnaires) \\
Absolute contraindications for CPET: fever, uncontrolled asthma, respira- \\
tory failure, acute myocarditis or pericarditis, uncontrolled arrhythmias \\
causing symptoms or haemodynamic compromise, uncontrolled \\
heart failure, acute pulmonary embolus or pulmonary infarction, and \\
children with mental impairment leading to inability to cooperate \\
Group 2: Children with any chronic disease, medical condition (cardiac, \\
neurologic, respiratory, muscular, or renal), or medical treatment and \\
those requiring any further specialized medical consultation \\
\hline
\end{tabular}

HRQoL studies among healthy controls and children with various chronic diseases [26-29].

In this study, three age versions of the PedsQL questionnaire will be used (e.g. 5-7, 8-12 and 13-17 years old). Parents and children will complete the QoL questionnaires during the routine paediatric cardiology consultation (inclusion visit). Children aged 8 years old and above will complete the PedsQL self-questionnaire under trained nurse supervision, as in our similar QoL studies [26-29].

\section{Secondary outcomes}

- The level of physical activity will be assessed by:

- The metabolic equivalent of task (MET), measured by a fitness tracker (ActiGraph GT3X accelerometer, Pensacola, FL, USA) [30]. The MET is defined as the ratio of physical activity to basal metabolic demand. The metabolic equivalence scale ranges from 0.9 MET (at rest) to 18 MET (run at $17.5 \mathrm{~km} / \mathrm{h}$ ). The fitness tracker will be assigned during the inclusion visit and the participants will be instructed to wear it at the waist at all times for 14 days, except during sleep and water-based activities such as swimming or bathing.

- The Ricci and Gagnon physical activity questionnaire, composed by 8 items (total score $<16$ points: no activity; 17 to 32 points: moderate activity; 33 to 40 points: intensive activity) will be completed during the inclusion visit by the participant. Children aged 8 years old and above will complete this questionnaire under trained nurse supervision [31].

- The EMAPS questionnaire (motivation scale for physical activity in a health context), a 18-item motivation scale towards health-oriented physical activity recently validated in French [32]. This questionnaire will be completed during the inclusion visit by the participant. Children aged 8 years old and above will complete this questionnaire under trained nurse supervision.

- The exercise capacity will be assessed by a cardiopulmonary exercise test (CPET), using the same methodology as in our previous paediatric CPET studies $[5,20,21,33]$. The following variables will be measured: maximum oxygen uptake $\left(\mathrm{VO}_{\text {max }}\right)$; ventilatory anaerobic threshold (VAT); ventilatory efficiency (VE/VCO2 slope), oxygen uptake efficiency slope (OUES), and maximum oxygen pulse $\left(\mathrm{VO} 2_{\max } /\right.$ maximum heart rate). Exercise test procedures in all participating laboratories will be harmonized before 
the start of the study. All centres will use the same CPET cycle ergometer protocol, to obtain a homogeneous incremental overall duration between 8 to 12 min: a 1-min rest; a 3-min warm-up (10 to 20 watts) in increments of 10,15 , or 20 watts each minute; a pedalling rate of 60 to 80 revolutions per minute; a 3-min active recovery (20 watts); and a 2-min rest. The CPET will be considered as maximal when 3 out of the 4 following criteria will be reached: respiratory exchange ratio $(\mathrm{RER}=\mathrm{VCO} 2 / \mathrm{VO} 2) \geq 1$, maximum heart rate $>85 \%$ of maximal age-predicted heart rate, limit of the patient's tolerance despite verbal encouragement, plateau of $\mathrm{VO} 2\left(\mathrm{VO} 2_{\max }\right)$ despite the increasing exercise intensity, and patient's inability to provide a minimum pedalling frequency of 60 per minute despite verbal encouragement. The VAT will be estimated using Beaver's method [34]. $\mathrm{VO}_{\max }$ and VAT values will be normalized in a percentage of the predicted $\mathrm{VO} 2_{\max }$ using reference values for cycle ergometer test in the general paediatric population $[35,36]$. When the $\mathrm{VO} 2_{\max }$ does not reach criteria for maximal effort, the peak VO2 will be informed, as usual in paediatrics $[37,38]$.

- The parents-reported QoL scores will be assessed by the proxy questionnaires of the PedsQL (e.g. 5-7, 8-12 and 13-17 y.o. proxy versions) [23]. Both parents will be able to participate and will therefore separately fill out a PedsQL proxy questionnaire.

All outcomes are reported in Table 2.

\section{Other data collection}

- Clinical outcomes: age at diagnosis (prenatal, postnatal), genetic anomaly, NYHA functional class, blood pressure, body mass index (BMI), healthcare usage (primary and secondary care contacts, hospitalisation), pacemaker, implantable cardioverter defibrillator (ICD), cardiac surgery, and medication.

- Socio-economic status of the patient's family.

- Safety outcomes.

\section{Sample size}

We aim to recruit a total of 214 children in both groups. The primary outcome is the difference in the selfreported QoL score with the PedsQL instrument between the children with inherited cardiac disorders and the control group. We used the results from our previous QoL cross-sectional studies in patients with $\mathrm{CHD}$ to calculate the sample size $[4,21,26-29,39]$. In our experience, as well as in similar studies using patient related outcomes, a difference of less than 5 points is not clinically relevant, and a difference of more than 10 points is ideal, but rarely achieved [2, 27, 29]. Therefore, we hypothesized to observe a difference in the total QoL score of $7 \pm 15$ points (over 100). With a $90 \%$ power, a bilateral alpha risk of $5 \%$, and potentially $10 \%$ of loss to follow-up or missing data on the primary outcome, we need to include 107 patients in the group 1 and 107 patients in the group 2.

\section{Statistical analysis}

Patients' characteristics will be presented using mean and standard deviation for continuous variables, and frequencies and proportions for categorical variables. QoL scores will be calculated with the parametric Student's $t$ test when the distribution was Gaussian and with the MannWhitney test otherwise. The effect size will be estimated using Cohen's d measure.

Association between QoL scores and physical activity, clinical and socio-demographic data will be studied using Pearson's or Spearman's coefficient. According to the

Table 2 Outcome measures

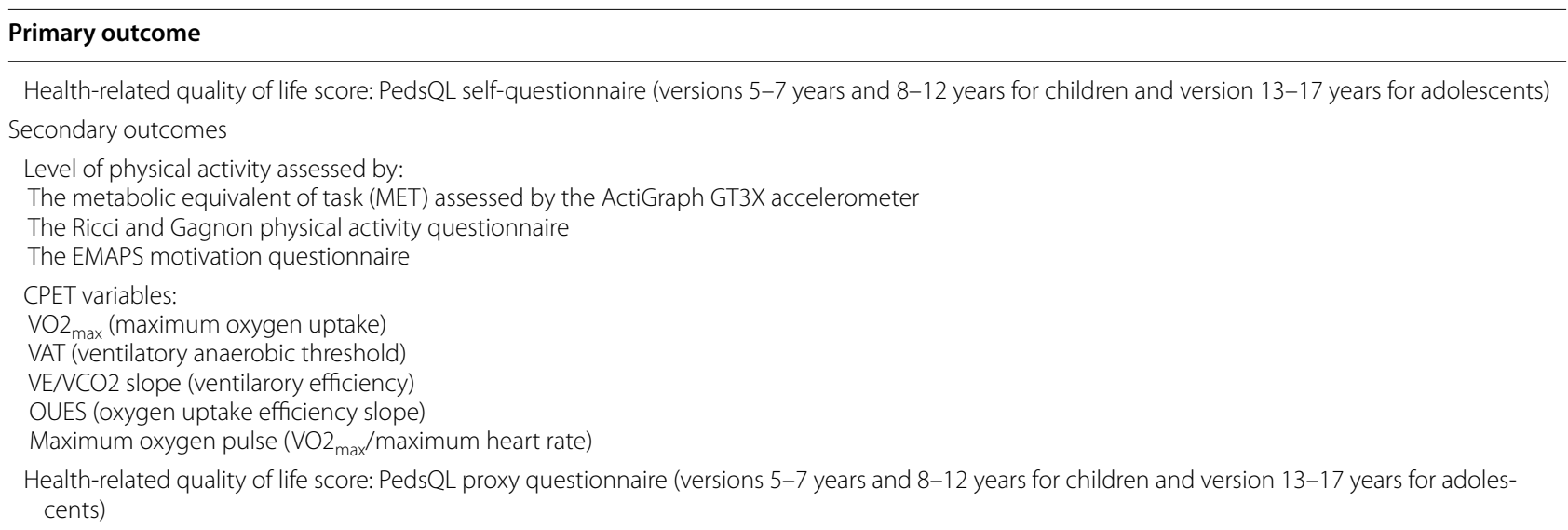


type of relation between QoL scores and these factors, a multiple linear regression or a generalized additive model (GAM) will be implemented for the different scores. In the GAM, a parametric portion of the model could be identified (with the vector of parameters associated with the set of explanatory variables). The non-parametric part of the model could be formed by smooth functions for clinical characteristics or age effects for example. One of the main advantages of the GAM implemented is the possibility to address the issue of linear dependency (i.e. collinearity) between these characteristics or age effects which often impedes their simultaneous estimation.

When considering multiple comparisons, the type I error will be adjusted.

The statistical significance will be set at 0.05 and analyses will be performed using Statistical Analysis Systems version 9 (SAS Institute, Cary, NC, USA). Data will be managed by the clinical research unit of Montpellier University Hospital, France, in collaboration with the paediatric and congenital cardiology department.

\section{Ethics}

The study will be conducted in compliance with the Good Clinical Practices protocol and Declaration of Helsinki principles. It was approved by a drawn national Ethics Committee (CPP Sud-Est VI, 2020-A00411-38). Informed consent will be obtained from all parents or legal guardians, and oral assent will be obtained from all children. The study was registered on Clinicaltrials.gov (NCT04712136).

\section{Expected results and perspectives}

In the continuity of our research program on QoL and physical activity in paediatric and congenital cardiology [4, 21, 22, 26, 26-29, 39], the QUALIMYOTYRHM trial should provide, for the first time, reliable data on health-related quality of life and physical activity in children with inherited cardiac arrhythmia or inherited cardiomyopathy. From our perspective, considering the QoL as the main outcome of this trial is both original and primordial in this young population with cardiac inherited disorders. Indeed, measuring patientreported outcomes (PRO) in paediatric cardiology should become a priority, considering that many young cardiac patients experience a low level of physical impairment during childhood, but an increased cardio-vascular risk during adulthood $[2,3,40]$. This is also in line with the European Medicines Agency (EMA) promotion of PRO measures in clinical research, and more and more drug trials, even in paediatrics, currently evaluate the quality of life as a secondary outcome [41]. Moreover, our results will contribute to provide consistent baseline clinical and QoL data for future interventional clinical trials in children with inherited cardiac arrhythmia or inherited cardiomyopathy.

The QUALIMYORYTHM trial also seeks to determine the level of physical activity in the contemporary generation of children with inherited cardiac disorders, using modern connected technology adapted to this population, such as fitness trackers. In the absence of any reliable risk stratification in those children, the current guidelines on eligibility of young athletes with inherited cardiac disorders for sports participation mainly rely on expert opinions [42-45], and conclusions by the experts have classically not been the same on both sides of the Atlantic [46]. The difference commonly made between recreational and competitive sport is probably more about medico-legal issues for the practitioner, than for the patient's own interest. For example, more than a decade ago, the European guidelines for competitive sport participation in the long QT syndrome were very restrictive [43]. Yet, in a recent study from a cohort of 172 appropriately managed children with long QT syndrome, cardiac event rates were low and occurred during recreational, but not competitive activities [47]. Similarly, in a cohort of 129 young athletes with ICD, most of them having a long QT syndrome or a hypertrophic cardiomyopathy, and participating in competitive or dangerous sports, there were no occurrences of death, arrest, or injury related to arrhythmia, during sports; interestingly, this study found that $27 \%$ patients received at least one shock, only 4 during sports, which is similar to unselected non-athletic paediatric ICD cohorts [48]. Based on convergent studies reporting a low risk of cardiac event in athletes with optimal treatment for long QT syndrome [47-50], a progressive trend towards a different medical approach has been observed, involving the patient-athlete in shared decision-making. Therefore, the 2015 US guidelines [51] have been less restrictive regarding high intensity recreational exercise and competitive sports, especially for asymptomatic genotype-positive/phenotype-negative athletes. More recently, the 2020 European guidelines have moved one step further in promoting supervised and regular physical activity in patients with inherited cardiac disorders [45].

Nevertheless, most published studies are retrospective and have not evaluated sports with the highest cardiovascular demand (only a few class IIIB and IIIC sports), or recreational sports in children with the rarest or most serious inherited cardiac disorders. Indeed, in their recent review, Masrur et al. concluded that there are insufficient data on the risks of exercise in Brugada syndrome to make recommendations for exercise [52]. Therefore, the QUALIMYORYTHM trial should 
contribute to precise how the recommendations are currently applied "in the real life", and measure their potential impact on QoL and physical activity in children with inherited cardiac arrhythmia or inherited cardiomyopathy.

Furthermore, the QUALIMYORYTHM trial will provide, for the first time in a population of children with inherited cardiac disorders, consistent cardiopulmonary fitness data, such as the maximum oxygen uptake $\left(\mathrm{VO} 2_{\max }\right)$, the anaerobic threshold, the ventilatory efficiency, and the exercise stroke volume. Moreover, this study will also bring original information about the level of motivation of children with inherited cardiac disorders for physical activity, and its degree of correlation with the actual levels of physical activity and exercise capacity. In paediatric cardiology, cardiopulmonary fitness has mainly been determined in children with CHD $[5,53]$, but these results cannot be easily transposed to inherited cardiac disorders, as the underlying pathophysiology and haemodynamic consequences are different. For instance, animal models have demonstrated that the beneficial effects of training on the heart effects of training are related to the signalling pathways of myocardial hypertrophy and fibrosis, but such findings need to be further analysed in patients with inherited cardiomyopathy [54].

If the QUALIMYORYTHM trial finds that physical activity and exercise capacity in children with inherited cardiac arrhythmia or inherited cardiomyopathy are similar to those of healthy children, without any additional risk, the level of evidence for future guidelines on sports eligibility in this population will be significantly improved. Conversely, if physical activity and exercise capacity in children with inherited cardiac disorders are significantly impaired, it will open a new research field on rehabilitation programs in this population. Indeed, if physical deconditioning is diagnosed and managed at an early stage in patients with inherited cardiac disorders, participation in rehabilitation programs could reverse the vicious circle of deconditioning, and, ultimately participate in reducing the cardio-vascular risks related to inactivity, as it has been suggested in CHD [22, 55-57], as well as in many chronic diseases [58].

\section{Study limitations}

The subjects in the control group will be recruited at the hospital and may not be considered as healthy as if they were recruited from the general population. However, this bias was limited in our previous similar studies, which showed very good correlations between QoL scores and $\mathrm{VO} 2_{\max }$ values with the respective normal values reported in the literature among the general population $[5,39]$.

\section{Abbreviations}

CHD: Congenital heart disease; CPET: Cardiopulmonary exercise test; QoL: Health-related quality of life.

\section{Acknowledgements}

The authors are grateful to CARDIOGEN for their support. We also thank Sophie Pierre and Lea Falloud, from the patient organisations "AMRYC" (association des patients atteints de maladies du rythme cardiaque), and "Ligue contre la cardiomyopathie", respectively, for their support.

\section{Authors' contributions}

Study concept and design: PA; Drafting of the manuscript: $\mathrm{PA}, \mathrm{AH}, \mathrm{OW}$; Critical revision of the manuscript for important intellectual content: all; Statistical analysis: MCP; Obtained funding: PA, OW; Administrative, technical, or material support: HA; Study supervision: PA. All authors read and approved the final manuscript

\section{Funding}

Montpellier university hospital is the sponsor of the QUALIMYORYHM trial. The study was funded after winning the 2019 call for proposal from the National Department of Health (DGOS-GIRCI-SOHO-APITHEM, www.girci-soho.fr) and the young researcher award from Montpellier University Hospital (AOI 2019). Additional research funding was obtained from the French Federation of Cardiology (2019 FFC"research team" call for proposal, www.fedecardio.org), and the national rare cardiovascular diseases network (2018 CARDIOGEN call for proposal, www.filiere-cardiogen.fr).

\section{Availability of data and materials}

Not applicable.

\section{Declarations}

\section{Ethics approval and consent to participate}

The study will be conducted in compliance with the Good Clinical Practices protocol and Declaration of Helsinki principles. It was approved by a drawn national Ethics Committee (CPP Sud-Est VI, 2020-A00411-38) and was registered on Clinicaltrials.gov (NCT04712136). Informed consent will be obtained from all parents or legal guardians, and oral assent will be obtained from all children.

\section{Consent for publication}

This study does not contain data from any individual person (images or videos).

\section{Competing interests}

The authors declare that they have no conflict of interests.

\section{Author details}

${ }^{1}$ Department of Paediatric and Adult Congenital Cardiology, M3C National Reference Centre, Haut-Lévêque Cardiology Hospital, Bordeaux University Hospital, Avenue de Magellan, 33604 Pessac Cedex, France. ${ }^{2}$ INSERM, Bordeaux Cardio-Thoracic Research Centre, U1045, University of Bordeaux, Pessac, France. ${ }^{3} \mathrm{HU}$ Liryc, Electrophysiology and Heart Modelling Institute, Fondation Bordeaux Université, Pessac, France. ${ }^{4}$ Paediatric and Congenital Cardiology Department, M3C Regional Reference CHD Centre, Montpellier University Hospital, Montpellier, France. ${ }^{5}$ Paediatric Cardiology and Rehabilitation Unit, Institut-Saint-Pierre, Palavas-Les-Flots, France. ${ }^{6}$ Epidemiology and Clinical Research Department, Montpellier University Hospital, Montpellier, France. ${ }^{7}$ Paediatric Cardiology and Physiology Department, Robert Debré University Hospital, University of Paris, AP-HP, Paris, France. ${ }^{8}$ Paediatric Cardiology Department, M3C Regional Reference Centre, Toulouse University Hospital, Toulouse, France. ${ }^{9}$ Department of Cardiology, National Reference Centre for Inherited Cardiomyopathy, University of Paris, AP-HP, Paris, France. ${ }^{10}$ Department of Cardiology, National Reference Centre for Inherited Cardiac Arrhythmia, L'institut du thorax, INSERM, CNRS, University of Nantes, Nantes University Hospital, Nantes, France. ${ }^{11}$ Department of Pediatric Cardiology and Pediatric Cardiac Surgery, L'Institut du Thorax, INSERM, CNRS, University of Nantes, Nantes University Hospital, Nantes, France. ${ }^{12}$ Department of Congenital Cardiology, National Reference Centre for Inherited Cardiac Arrhythmia, University of Lyon, Lyon University Hospital, Lyon, France. ${ }^{13}$ Paediatric Cardiology Department, 
Necker-Enfants malades, M3C National Reference Centre, University of Paris, AP-HP, Paris, France. ${ }^{14}$ Cardiology Department of Cardiology, Regional Reference Centre for Inherited Cardiac Arrhythmia, Montpellier University Hospital, Montpellier, France. ${ }^{15}$ PhyMedExp, INSERM, CNRS, University of Montpellier, Montpellier, France.

Received: 10 April 2021 Accepted: 18 July 2021

Published online: 28 July 2021

\section{References}

1. Spector LG, Menk JS, Knight JH, McCracken C, Thomas AS, Vinocur JM, et al. Trends in long-term mortality after congenital heart surgery. J Am Coll Cardiol. 2018;71:2434-46

2. Anker SD, Agewall S, Borggrefe M, Calvert M, Jaime Caro J, Cowie MR, et al. The importance of patient-reported outcomes: a call for their comprehensive integration in cardiovascular clinical trials. Eur Heart J. 2014;35:2001-9

3. Moons P, Kovacs AH, Luyckx K, Thomet C, Budts W, Enomoto J, et al. Patient-reported outcomes in adults with congenital heart disease: Intercountry variation, standard of living and healthcare system factors. Int J Cardiol. 2018;251:34-41.

4. Amedro P, Picot MC, Moniotte S, Dorka R, Bertet H, Guillaumont S, et al. Correlation between cardio-pulmonary exercise test variables and health-related quality of life among children with congenital heart diseases. Int J Cardiol. 2016;203:1052-60.

5. Amedro P, Gavotto A, Guillaumont S, Bertet H, Vincenti M, De La Villeon G, et al. Cardiopulmonary fitness in children with congenital heart diseases versus healthy children. Heart Br Card Soc. 2018;104:1026-36.

6. Takken T, Giardini A, Reybrouck T, Gewillig M, Hövels-Gürich HH, Longmuir PE, et al. Recommendations for physical activity, recreation sport, and exercise training in paediatric patients with congenital heart disease: a report from the Exercise, Basic \& Translational Research Section of the European Association of Cardiovascular Prevention and Rehabilitation, the European Congenital Heart and Lung Exercise Group, and the Association for European Paediatric Cardiology. Eur J Prev Cardiol. 2012;19:1034-65.

7. Longmuir PE, Brothers JA, de Ferranti SD, Hayman LL, Van Hare GF, Matherne GP, et al. Promotion of physical activity for children and adults with congenital heart disease: a scientific statement from the American Heart Association. Circulation. 2013:127:2147-59.

8. Budts W, Pieles GE, Roos-Hesselink JW, Sanz de la Garza M, D'Ascenzi F, Giannakoulas $\mathrm{G}$, et al. Recommendations for participation in competitive sport in adolescent and adult athletes with Congenital Heart Disease (CHD): position statement of the Sports Cardiology \& Exercise Section of the European Association of Preventive Cardiology (EAPC), the European Society of Cardiology (ESC) Working Group on Adult Congenital Heart Disease and the Sports Cardiology, Physical Activity and Prevention Working Group of the Association for European Paediatric and Congenital Cardiology (AEPC). Eur Heart J. 2020:41:4191-9.

9. Moola F, Fusco C, Kirsh JA. The perceptions of caregivers toward physical activity and health in youth with congenital heart disease. Qual Health Res. 2011;21:278-91.

10. Papasavvas T, Alhashemi M, Micklewright D. Association between depressive symptoms and exercise capacity in patients with heart disease: a meta-analysis. J Cardiopulm Rehabil Prev. 2017:37:239-49.

11. Karsenty C, Maury P, Blot-Souletie N, Ladouceur M, Leobon B, Senac V, et al. The medical history of adults with complex congenital heart disease affects their social development and professional activity. Arch Cardiovasc Dis. 2015;108:589-97.

12. Tamayo C, Manlhiot C, Patterson K, Lalani S, McCrindle BW. Longitudinal evaluation of the prevalence of overweight/obesity in children with congenital heart disease. Can J Cardiol. 2015;31:117-23.

13. Moola F, McCrindle BW, Longmuir PE. Physical activity participation in youth with surgically corrected congenital heart disease: Devising guidelines so Johnny can participate. Paediatr Child Health. 2009;14:167-70.

14. Czosek RJ, Kaltman JR, Cassedy AE, Shah MJ, Vetter VL, Tanel RE, et al. Quality of life of pediatric patients with long QT syndrome. Am J Cardiol. 2016:117:605-10.
15. Sleeper LA, Towbin JA, Colan SD, Hsu D, Orav EJ, Lemler MS, et al. HealthRelated Quality of Life and Functional Status Are Associated with Cardiac Status and Clinical Outcome in Children with Cardiomyopathy. J Pediatr. 2016;170:173-180.e1-4

16. Gow RM, Borghese MM, Honeywell CR, Colley RC. Activity intensity during free-living activities in children and adolescents with inherited arrhythmia syndromes: assessment by combined accelerometer and heart rate monitor. Circ Arrhythm Electrophysiol. 2013;6:939-45.

17. Hamang A, Eide GE, Nordin K, Rokne B, Bjorvatn C, Øyen N. Health status in patients at risk of inherited arrhythmias and sudden unexpected death compared to the general population. BMC Med Genet. 2010;11:27.

18. Rhodes AC, Murray B, Tichnell C, James CA, Calkins H, Sears SF. Quality of life metrics in arrhythmogenic right ventricular cardiomyopathy patients: the impact of age, shock and sex. Int J Cardiol. 2017;248:216-20.

19. Christian S, Somerville M, Taylor S, Spence JC, Giuffre M, Atallah J. The impact of physical activity modification on the well-being of a cohort of children with an inherited arrhythmia or cardiomyopathy. Cardiol Young. 2020;30:692-7.

20. Gavotto A, Vandenberghe D, Abassi H, Huguet H, Macioce V, Picot M-C, et al. Oxygen uptake efficiency slope: A reliable surrogate parameter for exercise capacity in healthy and cardiac children? Arch Dis Child. 2020;105:1167-74.

21. Abassi H, Gavotto A, Picot MC, Bertet H, Matecki S, Guillaumont S, et al. Impaired pulmonary function and its association with clinical outcomes, exercise capacity and quality of life in children with congenital heart disease. Int J Cardiol. 2019;285:86-92.

22. Amedro P, Gavotto A, Legendre A, Lavastre K, Bredy C, De La Villeon G, et al. Impact of a centre and home-based cardiac rehabilitation program on the quality of life of teenagers and young adults with congenital heart disease: The QUALI-REHAB study rationale, design and methods. Int J Cardiol. 2019:283:112-8.

23. Varni JW, Seid M, Kurtin PS. PedsQL 4.0: reliability and validity of the Pediatric Quality of Life Inventory version 40 generic core scales in healthy and patient populations. Med Care. 2001;39:800-12.

24. Tessier S, Vuillemin A, Lemelle J-L, Briançon S. Propriétés psychométriques du questionnaire générique français Pediatric Quality of Life Inventory Version 4.0 (PedsQLTM 4.0). Eur Rev Appl Psychol. 2009;59:291-300.

25. Amedro $P$, Huguet $H$, Macioce V, Dorka R, Auer A, Guillaumont $\mathrm{S}$, et al. Psychometric validation of the French self and proxy versions of the PedsQLTM 4.0 generic health-related quality of life questionnaire for 8-12 year-old children. Health Qual Life Outcomes. 2021;19:75.

26. Amedro P, Tahhan N, Bertet H, Jeandel C, Guillaumont S, Mura T, et al. Health-related quality of life among children with Turner syndrome: controlled cross-sectional study. J Pediatr Endocrinol Metab JPEM. 2017;30:863-8

27. Amedro P, Bajolle F, Bertet H, Cheurfi R, Lasne D, Nogue E, et al. Quality of life in children participating in a non-selective INR self-monitoring VKAeducation programme. Arch Cardiovasc Dis. 2018;111:180-8.

28. Abassi H, Bajolle F, Werner O, Auer A, Marquina A, Mura T, et al. Healthrelated quality of life correlates with time in therapeutic range in children on anticoagulants with International Normalised Ratio self-monitoring. Arch Cardiovasc Dis. 2020;113(12):811-20.

29 Abassi H, Huguet H, Picot M-C, Vincenti M, Guillaumont S, Auer A, et al. Health-related quality of life in children with congenital heart disease aged 5 to 7 years: a multicentre controlled cross-sectional study. Health Qual Life Outcomes. 2020;18:366.

30. Cunningham C, Spence JC, Stearns JA, Carson V, Kantor PF, Urschel S, et al Self-reported and accelerometer-measured physical activity in children with cardiomyopathy. J Cardiovasc Nurs. 2020;35:300-6.

31. Vuillemin A, Denis $G$, Guillemin F, Jeandel C. A review of evaluation questionnaires for physical activity. Rev Epidemiol Sante Publique. 1998:46:49-55

32. Boiché J, Gourlan M, Trouilloud D, Sarrazin P. Development and validation of the "Echelle de Motivation envers l'Activité Physique en contexte de Santé": a motivation scale towards health-oriented physical activity in French. J Health Psychol. 2019;24:386-96.

33. Gavotto A, Huguet H, Picot M-C, Guillaumont S, Matecki S, Amedro P. The $V$ e/ $\vee$ co2 slope: a useful tool to evaluate the physiological status of children with congenital heart disease. J Appl Physiol Bethesda Md. 1985:2020(129):1102-10. 
34. Beaver WL, Wasserman K, Whipp BJ. A new method for detecting anaerobic threshold by gas exchange. J Appl Physiol Bethesda Md. 1985;1986(60):2020-7.

35. Cooper DM, Berry C, Lamarra N, Wasserman K. Kinetics of oxygen uptake and heart rate at onset of exercise in children. J Appl Physiol Bethesda Md. 1985;1985(59):211-7.

36. Cooper DM, Weiler-Ravell D, Whipp BJ, Wasserman K. Aerobic parameters of exercise as a function of body size during growth in children. J Appl Physiol. 1984;56:628-34.

37. Barker AR, Williams CA, Jones AM, Armstrong N. Establishing maximal oxygen uptake in young people during a ramp cycle test to exhaustion. Br J Sports Med. 2011;45:498-503.

38. Rowland TW, Cunningham LN. Oxygen uptake plateau during maximal treadmill exercise in children. Chest. 1992;101:485-9.

39. Amedro P, Dorka R, Moniotte S, Guillaumont S, Fraisse A, Kreitmann $B$, et al. Quality of life of children with congenital heart diseases: a multicenter controlled cross-sectional study. Pediatr Cardiol. 2015;36:1588-601.

40. Saha P, Potiny P, Rigdon J, Morello M, Tcheandjieu C, Romfh A, et al. Substantial cardiovascular morbidity in adults with lower-complexity congenital heart disease. Circulation. 2019;139:1889-99.

41. Apolone G, De Carli G, Brunetti M, Garattini S. Health-related quality of life (HR-QOL) and regulatory issues. An assessment of the European Agency for the Evaluation of Medicinal Products (EMEA) recommendations on the use of HR-QOL measures in drug approval. Pharmacoeconomics. 2001;19:187-95.

42. Maron BJ, Chaitman BR, Ackerman MJ, Bayés de Luna A, Corrado D, Crosson JE, et al. Recommendations for physical activity and recreational sports participation for young patients with genetic cardiovascular diseases. Circulation. 2004;109:2807-16.

43. Pelliccia A, Fagard R, Bjørnstad HH, Anastassakis A, Arbustini E, Assanelli D, et al. Recommendations for competitive sports participation in athletes with cardiovascular disease: a consensus document from the Study Group of Sports Cardiology of the Working Group of Cardiac Rehabilitation and Exercise Physiology and the Working Group of Myocardial and Pericardial Diseases of the European Society of Cardiology. Eur Heart J. 2005;26:1422-45

44. Priori SG, Blomström-Lundqvist C, Mazzanti A, Blom N, Borggrefe M, Camm J, et al. 2015 ESC Guidelines for the management of patients with ventricular arrhythmias and the prevention of sudden cardiac death: The Task Force for the Management of Patients with Ventricular Arrhythmias and the Prevention of Sudden Cardiac Death of the European Society of Cardiology (ESC). Endorsed by: Association for European Paediatric and Congenital Cardiology (AEPC). Eur Heart J. 2015;36:2793-867.

45. Pelliccia A, Sharma S, Gati S, Bäck M, Börjesson M, Caselli S, et al. 2020 ESC Guidelines on sports cardiology and exercise in patients with cardiovascular disease. Eur Heart J. 2021;42(1):17-96
46. Schnell F, Behar N, Carré F. Long-QT syndrome and competitive sports. Arrhythmia Electrophysiol Rev. 2018;7:187-92.

47. Chambers KD, Beausejour Ladouceur V, Alexander ME, Hylind RJ, Bevilacqua L, Mah DY, et al. Cardiac Events During Competitive, Recreational, and Daily Activities in Children and Adolescents With Long QT Syndrome. J Am Heart Assoc. 2017;6(9):e005445

48. Saarel EV, Law I, Berul Cl, Ackerman M, Kanter RJ, Sanatani S, et al. Safety of Sports for young patients with implantable cardioverter-defibrillators. Circ Arrhythm Electrophysiol. 2018;11:e066305.

49. Aziz PF, Saarel EV. Sports participation in long QT syndrome. Cardiol Young. 2017;27:S43-8.

50. Johnson JN, Ackerman MJ. Return to play? Athletes with congenital long QT syndrome. Br J Sports Med. 2013;47:28-33.

51. Ackerman MJ, Zipes DP, Kovacs RJ, Maron BJ. Eligibility and disqualification recommendations for competitive athletes with cardiovascular abnormalities: task force 10: the cardiac channelopathies: a scientific statement from the American Heart Association and American College of Cardiology. J Am Coll Cardiol. 2015;66:2424-8.

52. Masrur S, Memon S, Thompson PD. Brugada syndrome, exercise, and exercise testing. Clin Cardiol. 2015;38:323-6.

53. Müller J, Böhm B, Semsch S, Oberhoffer R, Hess J, Hager A. Currently, children with congenital heart disease are not limited in their submaximal exercise performance. Eur J Cardio-Thorac Surg. 2013;43:1096-100.

54. Kyselovič J, Leddy JJ. Cardiac fibrosis: the beneficial effects of exercise in cardiac fibrosis. Adv Exp Med Biol. 2017;999:257-68.

55. Rhodes J, Curran TJ, Camil L, Rabideau N, Fulton DR, Gauthier NS, et al. Sustained effects of cardiac rehabilitation in children with serious congenital heart disease. Pediatrics. 2006;118:e586-593.

56. Gomes-Neto M, Saquetto MB, da Silva e Silva CM, Conceição CS, Carvalho VO. Impact of Exercise Training in Aerobic Capacity and Pulmonary Function in Children and Adolescents After Congenital Heart Disease Surgery: A Systematic Review with Meta-analysis. Pediatr Cardiol. 2016;37:217-24.

57. Amedro P, Gavotto A, Bredy C, Guillaumont S. Cardiac rehabilitation for children and adults with congenital heart disease. Presse Medicale Paris Fr. 1983;2017(46):530-7.

58. Booth FW, Roberts CK, Laye MJ. Lack of exercise is a major cause of chronic diseases. Compr Physiol. 2012;2:1143-211.

\section{Publisher's Note}

Springer Nature remains neutral with regard to jurisdictional claims in published maps and institutional affiliations.
Ready to submit your research? Choose BMC and benefit from:

- fast, convenient online submission

- thorough peer review by experienced researchers in your field

- rapid publication on acceptance

- support for research data, including large and complex data types

- gold Open Access which fosters wider collaboration and increased citations

- maximum visibility for your research: over 100M website views per year

At BMC, research is always in progress.

Learn more biomedcentral.com/submissions 